\title{
Management integration framework in a Shop- Floor employing self-Contained Assembly Unit for optoelectronic products
}

\author{
Maurizio Fiasché, Senior, IEEE, Gaia Ripamonti, Francesco G. Sisca, Marco Taisch, and Anna A. \\ Valente, Member, IEEE
}

\begin{abstract}
To establish practices and technological environments for using automated production systems in manufacturing of optoelectronic products, with characteristics of high customization of final products, of low volumes, make to order and especially of high manual activity is a challenge crucial for the future of manufacturing in Europe for this manufacturing sector. White' $R$ is an FP7-FoF-EU Project where the main goal is to design and produce a modular- adaptive - self-containedreconfigurable robotic island in a white room for production of two different types of optoelectronic products with features described above: laser diodes and solar cells. In this paper the production planning, scheduling and control issues of white' $R$ for HMLV optoelectronics is discussed and a management integration framework is proposed. The management framework presented in this work aims to sustain any company employing a self-contained automated and re-configurable robotic assembly island to organize and unify the development of its manufacturing capabilities still guaranteeing the alignment with the organizational strategy developed at the highest level of an organization. Two real cases are presented but the framework is applicable to any company in the sector analyzed. This is the first paper with application of a general framework for HMLV products in a white room based, self-contained highly automated assembly environment.
\end{abstract}

Index Terms - Production Planning, Production Scheduling, Management Integration Framework, Robotic Modular Systems, Re-configurable system, self-contained robotic island, HMLV

\section{INTRODUCTION}

$\mathrm{T}$ HE European manufacturing systems nowadays are driven towards the deployment of reconfigurable machinery and robots in order to support mass customized highly personalized products and fast reactions to variability of market demands è $[1,2]$. Despite this trend, in optoelectronic

Submitted on the $25^{\text {th }}$ of May 2015. This work was supported by the white'R Project, Grant FP7- FoF.NMP.2013 number 609228.

M. Fiasché, G. Ripamonti, F. G. Sisca, M. Taisch are with Politecnico di Milano, Department of Management, Economics and Industrial Engineering, Milano, Italy (e-mail: maurizio.fiasche@polimi.it, gaia.ripamonti@polimi.it, francescogiovanni.sisca@polimi.it,marco.taisch@polimi.it).

A. A. Valente is with the University of Applied Sciences and Arts of Southern Switzerland, Institute of Systems and Technologies for Sustainable Production, SUPSI-ISTePS, Manno, Switzerland (e-mail: anna.valente@supsi.ch). market - integrating electronics and optic devices - high handy level is still the main drive in manufacturing activities. The current Optoelectronic market is highly fragmented in small lot sizes, fast changing needs and high variety of products [3]. In literature, products with the above-mentioned characteristics are addressed to with the acronym HMLV that stands for High Mix Low Volumes. In HMLV production systems variety can be as high as 600 different products or greater, and incoming order quantities may range anywhere from 0 to 1000 for individual products within the mix over a particular time horizon [1]. Optoelectronic products are extremely varied and range from high volumes standard solutions to low volumes and highly variable solutions often designed on demand based on the specific user requirements. In this paper hence, we focus on the HMLV optoelectronics rather than the mass-produced ones (e.g. PCB). The realization of customized optoelectronic solutions is characterized by a high rate of complexity: products with extremely complex shape and made of fragile and delicate materials; highly product degradability; high number of product variants in relation to product geometry, technological features and performance ranges; frequent product evolution over time to match the market dynamics; low production volumes. These characteristics suggest that novel production systems able to provide comprehensive automated assembly operations, overall better saturation, reduced set-up and ramp-up times and equipment reusability are needed. Eventually the utilization of automated industrial equipment whose flexibility would be capable of matching the various production requirements across their lifecycles would globally impact on the reduction of cost for the products and the production while ensuring an increased agility towards the introduction of new product versions. The context of this paper is the white'R FP7 European Project, which develops a highly automated, selfcontained, white room based, multi-robotic island designed for the motion, assembly, and dis-assembly of optoelectronic products. The physical outcome of the project is the production of two demonstrators where the white' $\mathrm{R}$ island will be configured differently to be used in two existing shop floors of two end-users dealing respectively with laser diodes processing and customized solar energy systems. However this new technological tool bring along the need to develop a 
management framework to sustain the integration of the island into an existing shop floor and to investigate models and techniques to solve the decisional problem of production planning and scheduling.

The objective of this paper hence is to describe the mechatronic architecture of such a self-contained island, the typical integration issues and to propose a management integration framework of this highly automate robotic assembly unit in a pre-existing previously highly manual shop floor producing HMLV optoelectronics. The framework of integration is solved through a fuzzy multi-objective linear programming and validated on two different use cases. The paper is organized as follow: In section 1 it is introduced the scope of the work and its targets. In section 2 it is discussed the state of the art in the optoelectronic market, the challenges in the sector of HMLV optoelectronics and in the robotic modular units manufacturing industry. In section 3 it is described the mechatronic (re)configuration of the selfcontained island developed in the European project white' $R$ while, in section 4 the steps and the challenges that the integration of a self-contained robotic island within a preexisting shop floor present are described. In section 5, a new management framework for the integration process it is described and discussed. In section 6 two fuzzy multiobjective linear programming are briefly presented for the solution of the hierarchical production planning decision problem contained within the framework described in the previous section. Finally, results, discussions for future investigation and conclusions are presented.

\section{STATE OF THE ART AND CHALLENGES OF THE PRODUCT}

\section{A. State of the art of robotic solutions for flexible manufacturing systems}

The manufacturing island white' $R$ whose process has been conceived to accomplish the production requirements while minimizing the layout dimensions is a compact and agile equipment whose re-configurability feature is given by modular robots.

Modular robots are conceived as the composition of multiple blocks with uniform interfaces allowing for the transfer of mechanical forces and torque, electrical power, and communication through the robot. Building blocks consist of some primary structural actuated unit and some additional specialized units such as grippers, vision systems and energy storage units [4].

The field of reconfigurable robots has seen significant progress over the last twenty years. The work by Stoy et al. [5] provides a comprehensive overview of the latest solutions and approaches related to self-reconfigurable robots while addressing the mechanical, control, configuration and functional monitoring challenges. Examples of major results in the field of reconfigurable robotics can be found in [6-8]. More recent works deal with nesting mechanisms for robot modules [9]; direct and inverse kinematics [10]; communication and control [11]; motion planning [12].

The adoption of modular and reconfigurable robots presents a number of advantages [4], such as:

1) Versatility: reconfigurable robotic systems are more adaptive than conventional systems. The ability to reconfigure allows a robot to disassemble and reassemble to form new morphologies that are better suited for new tasks.

2) Robustness: since robot parts are interchangeable, it can also replace faulty parts autonomously, thus leading to selfrepair behaviors.

3) Low Cost: reconfigurable robotic systems can potentially lower overall robot cost by making many copies of one type of modules so economies of scale and mass production come into play. In addition, a range of complex solutions can be made from one set of modules, saving costs through reuse.

In manufacturing, these features result essential for users of robotic solutions producing very different families of products for several clients. Relying upon one or more reconfigurable robots could represent a major efficiency leverage, as it would allow fast and cost-effectively reaction to short-term changes coming from clients. The current practice associated to the need to accomplish future (forecasted) changes of production requirements is fulfilled by oversized robotic structures and, consequently, noticeably increasing the related investment costs.

However, these three advantages have not yet been fully implemented in reality and represent the main reasons for a limited diffusion of re-configurability for industrial robots adopted in manufacturing. In addition, the possibility to change the degrees of freedom make modular robots more versatile in their potential capabilities, but implies the risk to incur a performance trade-off and increased mechanical and control complexities. In fact, existing examples of reconfigurable robots are frequently constituted by a set of almost identical modules and any possible change of reaching and flexibility is managed by adding or subtracting these modules; this practice would be poorly viable in industrial robotics as it would boost the risk to affect accuracy of the manipulator and making more complex the dynamic control of the structure. In manufacturing, the possibility to have several families of robotic modules with very different shape, size and functionalities would be even instrumental dependently to the tasks to be accomplished and the modules' position in the kinematic chain. Examples of currently available research in this field is outlined in [13] with preliminary industrial prototypes found in [14].

Thus, the open questions the research and industry are facing for this category of reconfigurable robots are:

i) How to guarantee robotic solutions able to operate efficiently and effectively to manufacture high quality products with minimum economical and resource losses;

ii) What is the flexibility of the robot required to change configuration and behavior starting from a finite set of 
modules, which permit to accomplish demand or technological evolutions of a family of products [15].

\section{B. Production Planning, Scheduling and Control state of the art under uncertainty}

The design of a planning, scheduling and control system to handle disturbing events is case-specific and hence, must be designed and optimized for a specific shop floor.

To deal with the production planning and scheduling decision problems in such a manufacturing environment employing re-configurable robots, it must take into account the variety and the unpredictability occurring internally (e.g. resource availability) and externally (e.g. demand volumes and technological characteristics of the products) the shop floor.

There are various types of uncertainties in real-life situations and several practical manufacturing problems such as variations in demand, breakdown of machines, variations in processing time, shuffling in order priorities, changes in product routings and interruption by new orders [16].

M.A.S. Monfared and J.B. Yang [17] discussed three levels of uncertainty related to a planning, scheduling and control three-layered production management system. On the the first level of uncertainty occur the planning disturbing events such as changes in job arrival pattern, shift in total demand, change in quality expectation, change in product mix, change in market share, introduction of substitute product; on the second level occur scheduling disturbing event such as new job arrival, change in due date, change in priority, change in quantity, change in product design, change in process required; and eventually Monfared and Yang pinpoint control disturbing events such as material late arrival, operation delay, varying processing time, quality rejection, resource deadlock/blockage, tool unavailable, process routing not ready, machine load instruction not ready, utility not supplied, machine breakdown.

The literature, reports two different approaches that have been the most widely adopted by researchers to solve Production Planning and Scheduling (PPS) decision problems under uncertain environment. These approaches include stochastic programming and fuzzy programming. Since stochastic programming model is often very complex in structure that make it limited to apply in practical problems the fuzzy programming techniques is considered to be more efficient in solving real PPS problems.

Fuzzy approach may be used for developing Hierarchical Production Planning, constituted by an Aggregate Production Planning (APP) and a Disaggregate Production Planning (DPP) [18, 19] besides for implementing an adaptive scheduling and control system [16].

Fuzzy programming technique may provide systematic solution for APP, DPP problems in an easier way then stochastic techniques but still guaranteeing a fast reaction to the changing environment.

\section{ROOBOTIC ISLAND WHITE'R CONFIGURATION}

The configuration of the robotic island such as of a generic manufacturing system results from a multitude of different criteria ranging from profit and risk to technology and efficiency while also addressing the impact of external uncertainty (e.g. demand volumes and technological characteristics of the products) and internal uncertainty (e.g. resource availability). A further dimension to be considered refers to the roles of system builder and integrator and the system user. The task of the system builder (being the system designer) is to identify a set of alternative system configurations matching the production requirements with different cost and performance. The system user evaluates the alternative system configurations and defines the timing of acquisition of the resources, thus planning its system life-cycle.

The first step refers to the island configuration and enables the identification of the island equipment set able to match the production requirements. The second step is the layout configuration where the selected equipment is physically allocated in the shop floor. The third step deals with the robot configuration problem, thus determining the types and number of links and joints to compose the robotic arm, which is supposed to serve in the white' $R$ island and interact with the other equipment. The steps of the approach are outlined in Fig. 1. The three steps are iterative until the best trade-off of productivity, investments and resource efficiency is achieved. The resulting solution will be a full resource selection and integration representing the best trade-off between the specific configuration targets.

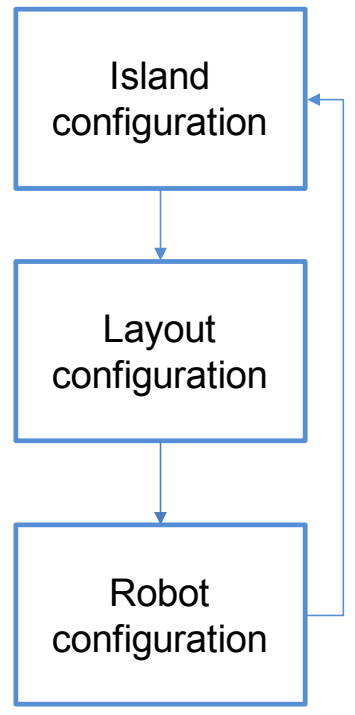

Fig. 1: Island configuration, selection of the resources model

The global benefits of the proposed approach is primarily related to the possibility to always determine the optimal 
solution of the problem in a very fast way by a computational point of view. Moreover, this will additionally permit to run the various steps both statically, during the green field decisions, as well as dynamically, when a change of production requirements must be accomplished via a reconfiguration.

\section{A. Mechatronic System configuration}

The manufacturing system design problem consists of selecting the best set of resources to satisfy the production requirements during the whole system lifecycle with the maximum expected profit. The considered resources are operational machines, transporters, load/unload stations, fixturing and storage. The considered operational machines are either performing technological processes such as soldering, gluing and mechanical assembly. Fixturing are assumed to be easily moveable among machines by proper automated handling systems and can be passive or active. The cost of the resources, which can be selected in the design phase, depends on their architecture and their performance. Every resource available to be selected has an efficiency impact indicator considering the resource carbon footprint, the age and its reliability over the time. The system design process can start from green field or can regard an existing production solution to be retrofitted to match new requirements (brown field design). Along with the set of selectable resources, the system designer needs information about the demand and the feasible process plans to manufacture the products. In particular, the demand is characterized by a set of products with their demand volumes and technological features. Volumes and products features can evolve over time, e.g. products may be modified, new products can be introduced and demand volume can change. Moreover, demand volumes can also be subject to mid-term variability. Short-term variability is considered at production management level. Information regarding the technological requirements of products to be manufactured determines the selection of resource types, while information regarding production volumes drives the choice of the number and productivity of resources.

\section{B. Layout configuration}

The resources selected as a result of the island configuration model need to be allocated in the space in a layout whose shape and dimensions should be the smallest possible in order to generate a very compact solution. The layout problem modeling has been widely investigated in research by utilizing different techniques and approaches. [18][19][20] report some examples of viable approached supporting the layout configuration. The focus in the proposed layout model is only related to the machine distribution in the layout as carriers and (un)load stations undergo functional constraints and cannot arbitrarily distributed by only focusing on the envelope space reduction. In fact, their allocation is considered once the machine layout has been decided.

The layout definition resulting from the proposed model will also determine the reaching dimensions for the robot that will operate in the island, i.e. the input for the robot configuration model.

The layout is modeled as a 2D Cartesian space as represented in Fig. 2. It shows only three machines as a simplified example; the reciprocal distance along $\mathrm{x}$ and $\mathrm{y}$-axes are illustrated for only Machine 1 and Machine 2.

The proposed model minimized the reciprocal distance between the machines whose envelope dimensions are available. It considers the part routing formulated as a physical distance every workpiece and the related fixturing are forced to execute in order for the fixture to complete its manufacturing process. The part routing is associated to the work plan.

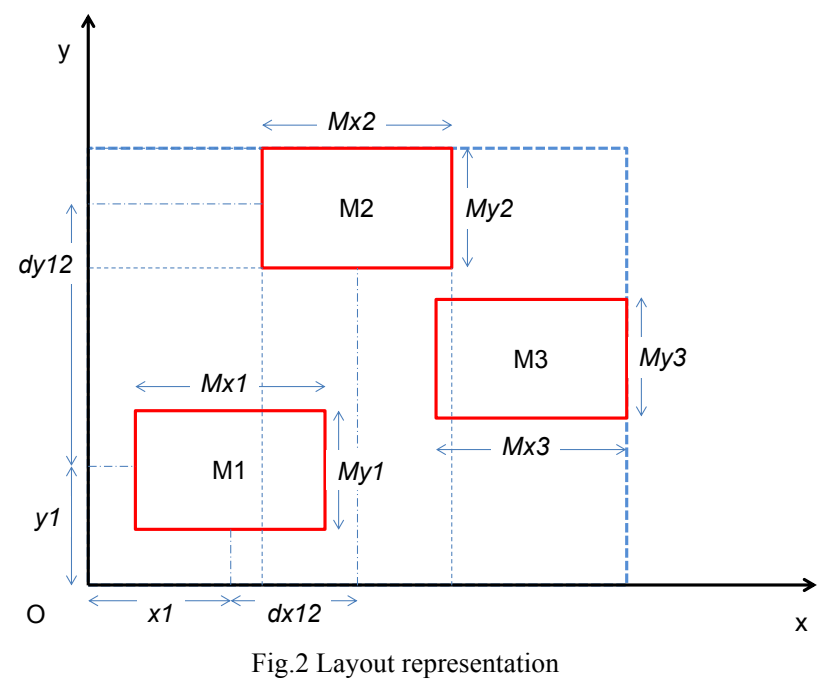

\section{The robot configuration}

The robot configuration objective is to generate a configuration synthesis of reconfigurable manipulator, i.e. selecting the type and number of joints and links to be nested to form the robot manipulator both considering the modules to be assembled in the robot first configuration and all the possible reconfigurations. The configuration problem addresses both kinematics and dynamics of the robotic structure and will be performed by addressing a number of design criteria, which have been listed in the following.

Industrial robots must present specific Key Performance indicators (KPIs) such as accuracy, high speed and low costs. Recently also the energy efficiency plays a major role in the robot assembly and management. This latter KPI is often addressed under the economic perspective when the operational costs are modeled. The KPI considered in the proposed configuration approach are listed in the following:

High efficiency in performing the considered manufacturing tasks over time, thus in exploiting working and not working movements while accomplishing the precision constraints. 
This KPI is measured as a task throughput (number of tasks per unit of time).

Low investment cost of the robot over the robot lifecycle (min configuration costs, min reconfiguration costs, min maintenance costs).

The configuration algorithm generates a number of configurations, which are analyzed kinematically, statically and dynamically until selecting the solution, which represents the best trade-off in accordance to the objective functions. The algorithm refers to a set of hypothesis, which has been listed in the following:

1) Hp1 serial robots.

2) Hp2 the position of the basis of the robot is assigned.

3) Hp3 every module presents one active input port and one active output port.

The robot configuration process is demanded to satisfy a number of constraints, which ensure the correct execution of the manufacturing tasks to be performed by the robot with accuracy, stiffness and reliability over the time.

The modeling of the robot constraints starts from the analysis of the robot tasks to be executed so that every production task is associated to one or more end-effectors and a set of robot tasks. Every task is associated to an ID and one or more end effectors capable of performing it. From the endeffector it can be derived the payload to be managed by the robot. The type of task and the end effectors permit to determine the minimum number of degree of freedom for the manipulator. Assuming the basis of the robot has a known and fixed position and the working area is assigned, all tasks constraint the minimum working area that should be covered by the robot. This information drives the selection of the robot reachability space. Every task is associated to a tolerance range, which puts boundaries to the accuracy of the working points for the robot. In fact, accuracy in exploiting the tasks meant as the gap between the reference target value and the actual value of the working points. The accuracy will be translated in error measure for translation and rotational in accordance to [21].

Every single module of the robot is associated to an impact index $\mathrm{p}$. This value embraces information about:

1) the module lifecycle formulated as an ageing parameter [tcurrent-tBoL]

2) the embedded energy consumption formulated as carbon footprint $\mathrm{CO} 2$

3) and the module MTTF representing the module reliability.

These three components of the impact index are leveraged by coefficients $\beta, \gamma, \eta$ whose value is defined positive $[0,1] \in$ $\mathrm{R}+$. These coefficients are set by the user in accordance to the importance of the specific aspect compared to the others.

The configuration approach will ensure the satisfaction of constraints governing the static behavior of the robot along with the dynamic one.
The static constraints refers to:

1) Satisfaction of the working space by properly selecting the reachability and dexterity space of the robot

2) Satisfaction of the min Degree of Freedom (DoF)

3) Realization of the task by respecting the task tolerances (static modeling)

The dynamic constraints refers to:

1) Robot stiffness and damping constraints

2) Robot accuracy considering moving components

3) Robot reliability (accuracy over the robot lifecycle)

4) Maximum allowable velocity, acceleration and torque at the end-effector and consequent ratios on the joints

IV. WHITE'R INTEGRATION CHALLENGES IN A PREEXISTING SHOP-FLOOR

In section III, the mechatronics aspects of robot (re)configuration in white' $\mathrm{R}$ have been presented. In this section, the focus is about the production management system of the typical machinery integrated in a pre-existing shop floor highly manual. Here below after presenting the typical characteristics of a shop floor suitable for the use of white'R, the peculiar challenges for the integration of the white' $R$ within the pre-existing shop floor are discussed.

\section{A. Typical Shop Floor}

The typical production Environment of a shop floor suitable for integration of a self-contained robotic island as the white' $R$ is a high handy rate flow shop where despite the fact that activities are carried on manually by operators the logical production phases are neat and scoped. Generally the machinery used in these production environments is most likely dedicated to one or few more operations and thus it is easy to be integrated with the white' $R$ island (hereafter called simply white'R). White'R can be simplified as a box where a storage of raw material is designed to grant a certain autonomy during a fixed time horizon and a set of specific assembly processes are designed and customized for each diverse application (Fig. 3).

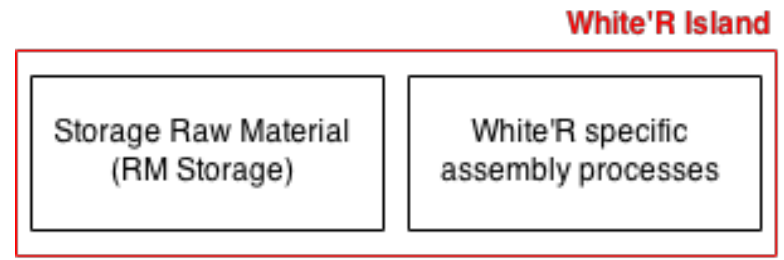

Fig. 3 - white'R island macro-functions

In both the end-users of white'R Project, the whole production cycle goes through automated activities intersected 
with manual activities. The shop floors are carried on by law populated work teams, whose operators' interoperability rate is high. The layout of the two plants is pretty contained, and develops on a single floor where both preparation of components and final assembly is carried on.

The products are basically rolled into one, starting from a range of components, which lay on few levels of the Bill Of Materials (BOM). It is desirable that the raw material storage of the shop floor and the white'R's one would be on the same level and easy to be connected each-others.

\section{B. The different integration scenarios}

The island is sat up in a way that the material flow goes along with the technological cycle. Consequently, from the preparation of raw material in the white' $R$ storage until the final assembly operations of the final product a flow shop is constituted. The flow shop may be internal white'R, or constituted by the island and one or more complement manual assembly processes (Fig. 4), downstream (a) or upstream (b), or both (c).

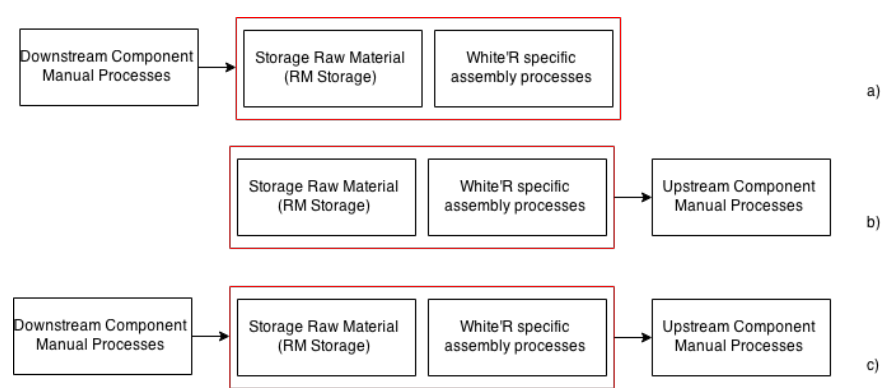

Fig.4 - The different integration scenarios

\section{Production Planning Integration issues}

In order to integrate a white' $R$ assembly island in an existing shop floor it is necessary to take into account several aspects arising from the production environment in terms of Production Planning and Control System (PPCS) too. The high level of customization of the optoelectronics to which is addressed the use of white' $R$ implies difficulties in both aggregate and disaggregate planning because of high uncertainty in the definition of the items to be planned to assembly over the time. The forecasting process is challenged by this character of variability and uncertainty over the mix of products demanded by the market. The commercial functions along with the production one need to track down wide clusters of family products in order to comprehend the greatest number as possible of varieties but still circumscribing them within certain generic features. This clusterization process aim to provide APP the most reliable demand forecast robust to demand level uncertainty.

The BOM, which is the input of the Material Planning, is also characterized by uncertainty present in demand forecasting. Indeed, the capacity for a client of configuring a customized product creates a multiple, nearly infinite, possible BOM. In parallel of the Material Planning (MP) is displayed the Master Production Scheduling (MPS).

Moreover, in a white'R employing production environment, the allocation of the production mix is founded on the white'R availability in term of weekly working shift and it is not allocated basing on the traditional sequencingscheduling problem. Since the components' variability in a white' $R$ product it is not defined, also the cycle time needed to create a product is not determined, thus also the white' $R$ productivity is not bordered in advance.

\section{The different capacity scenarios}

According with the different shop floors scenarios there are corresponding different shop floors capacity scenarios.

In the specific production environment after the integration of the self-contained island the main concern that has been scoped, is to understand how the assembly line capacity is given.

In the wider scenario, white'R may be adopted by a company to increase its Assembly capacity whilst still keeping a manual assembly area for some phases within the whole assembly process. The white'R may include whole assembly phase or just carry out a certain flow of assembly phases. In this latter case, the capacity of the assembly line is equal to the bottleneck's capacity value:

$C_{\text {ass.shoflloor }}=C_{\min }=T c_{l j} \cdot[p c s / h r s] * A v_{l j}[h r s /$ day $]$

Where $\mathrm{Tc}_{\mathrm{lj}}$ is the cycle time to assemble an item $\mathrm{j}$ in the assembly line 1 . Where $1=w r A L$ or $1=m A L$ if the line considered is respectively white'R-employing Assembly line (wrAL) or Manual Assembly Line (mAL).

Another scenario is to use white' $R$ in order to replace all the assembly phases previously carried on by manual intervention. In this case, the capacity of the shop floor is equal to the capacity of white'R:

$C_{\text {ass.shoflloor }}=C_{w r A L}$

In another scenario, the two capacities concur to create the overall Shop floor capacity assembling the same family product thus:

$C_{\text {ass.shoflloor }}=C_{m a A L}+C_{w e A L}$

Another scenario is that where the white' $\mathrm{R}$ is dedicated to a specific family of products while a manual assembly line assemble the products not assembled by white'R:

$$
\begin{aligned}
& \sum_{j=1}^{m-1} c=C_{m a A L} \\
& \sum_{j=m}^{n} c=C_{w r A L} \\
& C_{\text {ass.shoflloor }}=C_{m a A L} J=1 \ldots(m-1)
\end{aligned}
$$


$C_{\text {ass.shoflloor }}=C_{\text {wrAL }} J=m \ldots n$

\section{E. Scheduling challenges}

Considering scheduling as a decision-making process used on a regular basis in order to allocate resources to tasks over given time periods and which goal is to optimize one or more objectives [24] the white'R Scheduling problem is not the typical one sequencing the jobs with their relatives due dates upon a certain amount of resources (machines).

Whilst the scheduling process may be developed with heuristic or metaheuristic techniques indiscriminately for the Manual Assembly Line, on the white'R Assembly Line the scheduling problem is more like a capacity planning over the shifts within a certain time horizon.

Since white' $R$ is a batch system, the real capacity of the white'R assembly line is given for a shift. After the specific item $\mathrm{j}$ to be produced in a shift is chosen, the corresponding quantity $q$ assembled in the same shift for the specific item $j$ is fixed to (Fig.2). It means that for each item $\mathrm{j}$ there is a specific white'R assembly line capacity, $\mathrm{C}_{\mathrm{wrALj}}$. Therefore, the scheduling problem in a production environment employing white' $R$ is to allocate its capacity on each shift within a certain time horizon $\mathrm{T}$ such to satisfy the due date for each item $\mathrm{j}$ $\left(d_{j}\right)$. The final capacity of the white'R assembly line is given by:

$$
\min \left(t_{\text {lavj }} * A V ; R_{j}\right)
$$

Where $t_{\text {lavj }}$ is the time needed to execute a manual intervention (upstream and/or downstream) complement to the white' $R$ cycle, Av is the availability of the specific resource and $R_{j}$ is the throughput of white' $R$ for the item $j$.

\section{THE MANAGEMENT INTEGRATION FRAMEWORK}

In this paragraph, it is presented two different versions of the integration framework: the first one is simple and the second one is a framework considering uncertainty.

\section{A. Plain}

The framework presented in Fig. 5 is a management integration framework with which a company employing a white'R may organize and unify the development of its manufacturing capabilities still guaranteeing the alignment with the organizational strategy developed at the highest level of an organization [1].

The Production Planning Process (PPP) starts after Business Planning Process (BPP), which lays on a higher level and provides all specific measurable objectives needed to achieve the mission of the company considered, and the demand forecast process (or the actual orders) received for a long time horizon, which is generally 5-6 years on time buckets of the year.
In this integration management framework, it is adopted the concept of Hierarchical Production Planning System (HPPS) presented by M.K. Omar et al. [22, 23]. Production Planning Process is composed by two hierarchical levels: the first considers aggregate production needs in terms of product family $i$ and wide time buckets, Aggregate Production Planning (APP) [22], the second considering each item $\mathrm{j}$ within the product family $\mathrm{i}$ along with shorter time buckets, Disaggregate Production Planning (DPP) [23].

Demand forecast is the variable taken as the input for the APP. APP process deals with matching assembly capacity to forecasted demand, varying customer orders over the medium term from 3 to 18 months. The demand forecasted is seen aggregated in product family (i) and the variables needed to be monitored are: storage of finish product (FP), capacity available of the assembly area of the Shop Floor (Cfp), which in the production environments considered coincides with the entire Shop Floor. 


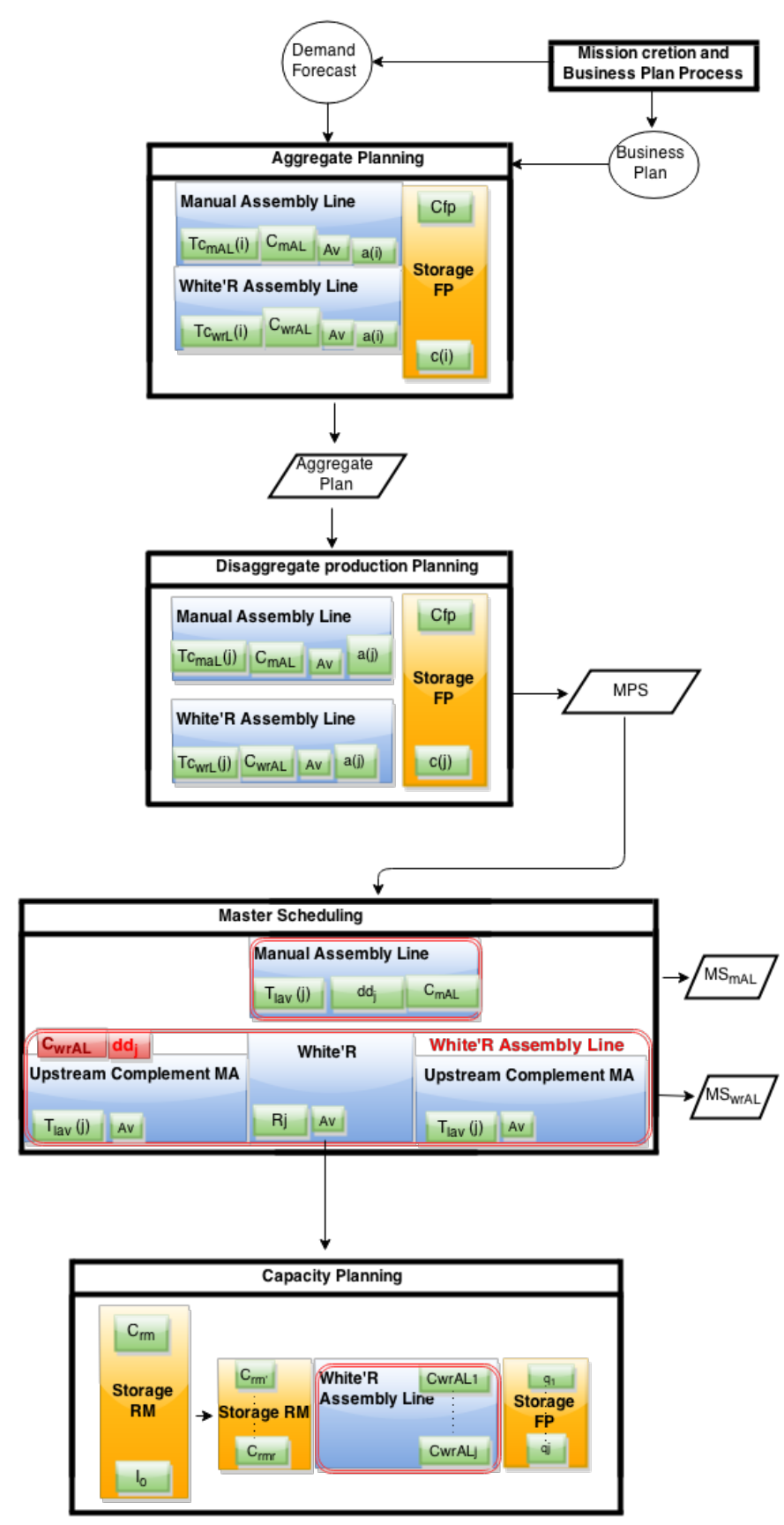

Fig. 5 - Management Integration Framework

The output of the APP, which is the amount of production for each aggregated family of product at the period $\mathrm{T}$ satisfying the capacity constraints and optimizing certain criteria [23], goes as input to the DPP, which is the second level of the entire PPP.

In order to link APP with DPP it is assumed true the equation:

$$
\sum_{j=1}^{J} \sum_{t=1}^{T} q_{j t}=q_{T i l} \forall i, l
$$

Where $\mathrm{q}_{\mathrm{tj} i \mathrm{l}}$ is the quantity assembled at time $\mathrm{t}$, for item $\mathrm{j}$ of the product family $\mathrm{i}$, in line 1 considered by the DPP while $\mathrm{q}_{\mathrm{Til}}$ is the production of the product family $i$ at aggregate time $T$ in line 1 considered by the APP.

The output of the DPP is the MPS, which is the amount of item $\mathrm{j}$ to be assembled by the whole Shop Floor at time $t$.

Both of the Planner we thought in our framework indicates costs to be considered for a generic case as set up costs a for assembly different family or different items at time $t, a_{i t}, a_{j t}$ and stock costs $\mathrm{c}_{\mathrm{it}}, \mathrm{c}_{\mathrm{jt}}$.

Upon these two levels specific Fuzzy Linear Programming models to solve the APP and DPP problems considering typical costs for a self-contained reconfigurable and robotic island as white'R, can be developed. The MPS enters the scheduler where both manual assembly line and white' $R$ assembly line are considered. The second one is constituted in the wider scenario of a line where upstream there may be still manual operations as well as in the downstream.

\section{B. With uncertainty control}

In this work, it is assumed that the uncertainty might occur on three different levels: $\Omega_{1}, \Omega_{2}, \Omega_{3}$ (Fig. 6).

In fact planning, scheduling and control functions can be seen into a hierarchical structure. It can therefore be considered as a collection of mechanisms for treating system dynamics or disturbances organized into a three level structure.

At the lower level the disturbance $\Omega_{1}$ occurs at the shop floor physical system level such as availability within white' $R$ assembly line and manual assembly line or real cycle times differing from nominal ones.

At the middle layer, the scheduler deals with uncertain order related events, $\Omega_{2}$.

Finally, at the higher layer of the hierarchy, the PP system is affected by uncertainties $\Omega_{3}$, which are long term disturbing events.

Therefore, $\Omega_{1}$ is uncertainty at the lower level, the level of shop floor, which impacts on Availability, Cycle Times and LTs. $\Omega_{2}$ is the uncertainty at the middle level, the level of orders, which impacts on the due dates (dd). $\Omega_{3}$ is the uncertainty at the higher level, the level of long term, which impacts on demand forecast and on BOM. 


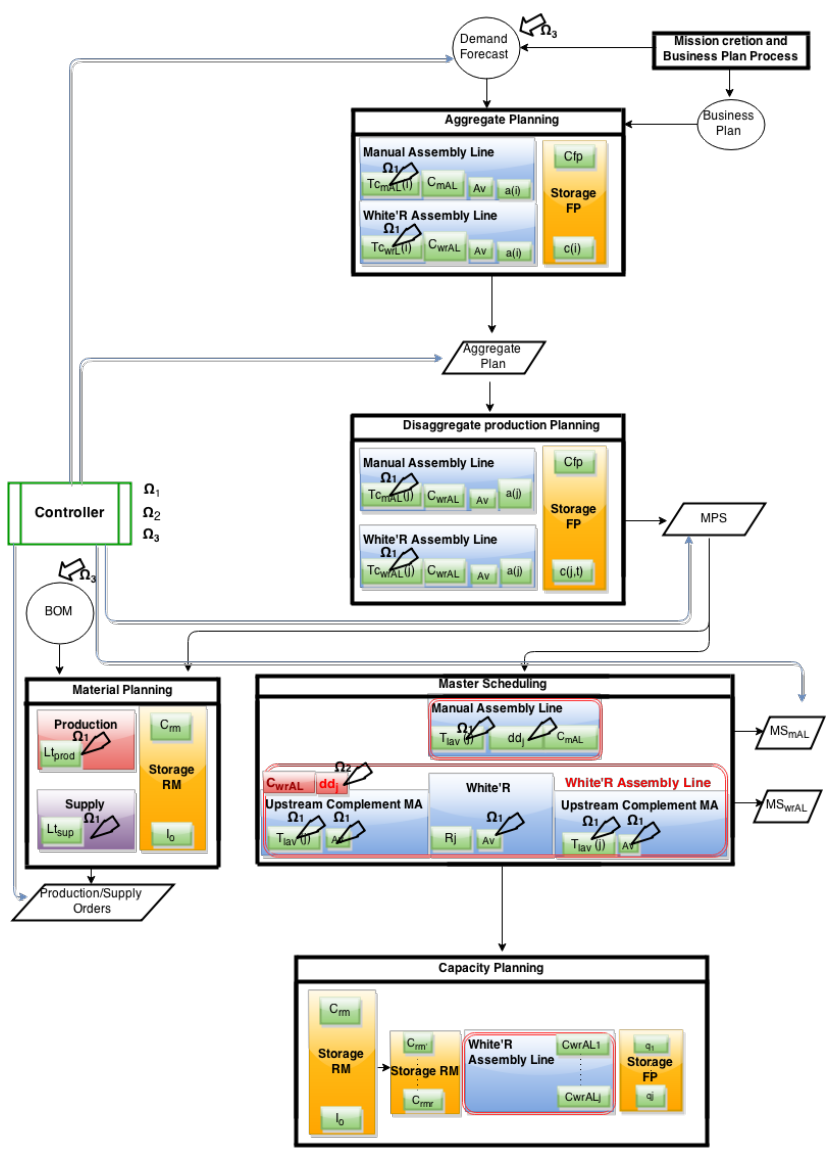

Fig. 6 Management Integration Framework with uncertainty control

\section{VI.FUZZY LINEAR PROGRAMMING MODELS FOR APP AND DPP}

In this section, the model formulation of aggregation and disaggregation levels in line with elaborating models in $[22,23]$ is presented.

The sign " $"$ " and " $"$ indicates the fuzzy constraint and parameter.

Indices

t period: $1, \ldots, \mathrm{T}$

i product family: $1, \ldots, \mathrm{N}$

1 line: $1, \ldots, \mathrm{L}$

j item: $1, \ldots, J$

\section{Parameters}

$\mathrm{q}_{\text {ilt }} \quad$ assembly level of product family $\mathrm{i}$ in line 1 in period $\mathrm{t}$

$\mathrm{q}_{\mathrm{j} j \mathrm{ilt}}$ production level of item $\mathrm{j}$ of product family $\mathrm{i}$ in line 1 in period $t$

$\mathrm{Z}_{\mathrm{il}} \quad$ unit assembly cost for product family $\mathrm{i}$ in line 1

$\mathrm{z}_{\mathrm{jil}} \quad$ unit assembly cost for item $\mathrm{j}$ of family $\mathrm{i}$ in line 1

$a_{i l} \quad$ set-up cost for family $i$ in line 1

$a_{j i l} \quad$ set-up cost for item $j$ of family $i$ in line 1

$c_{i} \quad$ unit inventory cost for product family $i$

$c_{\mathrm{ji}} \quad$ unit inventory cost for item $\mathrm{j}$ of family $\mathrm{i}$

$\mathrm{st}_{\mathrm{il}} \quad$ number of set ups for family $i$ in line 1

st $_{j i l} \quad$ number of set ups for item $j$ of family $i$ in line 1

$\mathrm{H}_{\mathrm{it}} \quad$ inventory level of product family $\mathrm{i}$ in period $t$

$\mathrm{H}_{\mathrm{jit}}$ inventory level of item $\mathrm{j}$ family $\mathrm{i}$ in period $\mathrm{t}$

Objective function for the APP

$$
\min \sum_{i=1}^{N} \sum_{l=m A L}^{w r A l} \sum_{t=1}^{T}\left(\hat{z}_{i l} * \hat{q}_{i l t}+\hat{a}_{i l} * s t_{i l}\right)+\sum_{i=1}^{N} \sum_{t=1}^{T} H_{i t} * \hat{c}_{i}
$$

Objective function for the DPP

$$
\min \sum_{j=1}^{J} \sum_{i=1}^{N} \sum_{l=m A L}^{w r A L} \sum_{t=1}^{T}\left(\hat{z}_{j i l} * \hat{q}_{j i l t}+\hat{a}_{j i l} * s t_{\mathrm{jil}}\right)+\sum_{j=1}^{J} \sum_{i=1}^{N} \sum_{t=1}^{T} H_{j i t} * \hat{c}_{i t}
$$

In order to link APP with DPP it is assumed true the equation:

$$
\sum_{j=1}^{J} \sum_{t=1}^{T} q_{j t} \cong q_{T i l} \forall i, l
$$

Where $\mathrm{q}_{\mathrm{tj} i \mathrm{l}}$ is the quantity assembled at time $\mathrm{t}$, for item $\mathrm{j}$ of the product family $i$, in line 1 considered by the DPP while $\mathrm{q}_{\mathrm{T}} \mathrm{il}$ is the production of the product family $\mathrm{i}$ at aggregate time $\mathrm{T}$ in line 1 considered by the APP.

The relative constraints and implementation of the definition of variable cost is under consideration for further work of research. 


\section{RESULTS AND CONCLUSIONS}

In this paper, the issue of how to switch from a highly manual production environment to a highly automated has been approached in a manufacturing environment of optoelectronic products with HMLV characteristics. Mechatronics aspects have been developed and described with a complete description of designing approach and finally the typical problems of integration of self-contained robotic assembly island such a white'R have been focused on hierarchical planning, scheduling and capacity issues. Different scenarios of the integration are available and corresponding capacity sizing issues come along.

An integration framework for supporting management has been proposed. Uncertainty is also to be considered in looking through the integration problem of a self-contained assembly unit such as white'R and different techniques for solving PPS problems are still needed to be investigated. Fuzzy Linear Multi-Objective Programming may be a suitable solution for both APP and DPP problems provided that properly defining costs to be minimized in such a shop floor using a white'R. In the future other adaptive approaches could be proposed [25][26][27], authors are investigating for possible innovative solutions.

\section{REFERENCES}

[1] R.M. Mahoney, "High-Mix Low-Volume", Hewlett Packard, 1997

[2] M. Mitsuishi, K. Ueda, F. Kimura, "Manufacturing Systems and Technologies for the New Frontier", The 4lst CIRP Conference on Manufacturing Systems, Tokio, Japan, May 26-28, 2008.

[3] B.W. Hueners, M. K. Formica, "Moving Toward Automation", Palomar Technologies 2013, http://www.photonics.com/EDU/Handbook.aspx?AID=25506

[4] M. Yim, W. Shen, B. Salemi, D. Rus, M. Moll, H. Lipson, E. Klavins, G. Chirikjian, "Modular self-reconfigurable robot systems," IEEE Robotics \& Automation Magazine, vol. 14, no. 1, pp. 43-52, 2007.

[5] K. Stoy, D. Brandt, D.J. Christensen, "Self-reconfigurable Robots, An introduction", The MIT Press, 2010.

[6] A. Lyder, R. Mendoza Garcia, K. Stoy, "Mechanical design of Odin, an extendable heterogeneous deformable modular robot," in Proc. IEEE/RSJ International Conference on Intelligent Robots and Systems, Nice, France, 2008, pp. 883-888.

[7] A. Sproevitz, A. Billard, P. Dillenbourg, A. Ijspeert, "Roombotsmechanical design of self reconfiguring modular robots for adaptive furniture," in Proc. IEEE International Conference on Robotics and Automation, Kobe, Japan, 2009, pp. 4259-4264.

[8] J. Davey, N. Kwok, M. Yim, "Emulating self-reconfigurable robots design of SMORES system," in Proc. IEEE/RSJ International Conference on Intelligent Robots and Systems, Vilamoura, Portugal, 2012, pp. 4464-4469.

[9] M.G. Catalano, G. Grioli, M. Garabini, F. Bonomo, M. Mancinit, N. Tsagarakis, A. Bicchi, "VSA-CubeBot: A modular variable stiffness platform for multiple degrees of freedom robots," in Proc. IEEE International Conference on Robotics and Automation, Shanghai, China, 2011, pp. 5090-5095.

[10] P. Xinan, W. Hongguang, J. Yong, Y. Cen, "Research on kinematics of modular reconfigurable robots," in Proc. IEEE International Conference on Cyber Technology in Automation, Control, and Intelligent Systems, Kunming, China, 2011, pp. 91-96.

[11] M. Hofbaur, M. Brandstotter, S. Jantscher, C. Schörghuber, "Modular re-configurable robot drives," in Proc. IEEE International Conference on Robotics Automation and Mechatronics, Singapore, 2010, pp. 150155.

[12] H. Feili, S. Wei-Min, "On the complexity of optimal reconfiguration planning for modular reconfigurable robots," in Proc. IEEE International Conference on Robotics and Automation, Anchorage, AK, 2010, pp. 2791-2796.

[13] J. Sulzer, I. Kovač, "Enhancement of positioning accuracy of industrial robots with a reconfigurable fine-positioning module," Precision Engineering, vol. 34, no. 2, pp. 201-217, 2010.

[14] www.modbot.com

[15] A. Valente, "Reconfigurable Industrial Robots - an integrated approach to design the joint and link modules and configure the robot manipulator". ASME ReMAR Conference 2015. 22-24 July, Beijin, China.

[16] M.A.S. Monfared, S.J. Steiner, "Fuzzy adaptive scheduling and control systems", 1998

[17] M.A.S. Monfared, J.B.Yang, "Design of integrated manufacturing planning, scheduling and control system: a new framework for automation", The International Journal of Advanced Manufacturing Technology, Vol. 33, Issue 5-6, pp 545-559. June, 2007.

[18] Shafigh F., Defersha F.M., Moussa S.E., "A. Comprehensive model for the design of distributed layouts in manufacturing systems", in Proc. Of the 2014 Industrial Systems Engineering Research Conference, Montreal, Canada, June, 2014

[19] Abdi M.R., Labib A.W., "A design strategy for Reconfigurable Manufacturing Systems (RMSs) using the Analytical hierarchical Process (AHP): a case study", International Journal of Production Research, Vols. Vol.41, No.10, pp. 2273-2299, 2003

[20] Zhao X. Wang J., Luo Z., "A stochastic model of a reconfigurable manufacturing syste,, Part1: A framework", International Journal of Production Research, Vols. Vol. 38, No. 10, pp. 2273-2285, 2000

[21] Bi Z.M. Zhang W.J., "Concurrent Optimal Design of Modular Robotic Configuration", Journal of Robotic Systems, Vols. Vol. 18, no.2, pp. 7787,2000

[22] M.K. Omar, M. M. Jusoh, M. Omar, "Investigating the Benefits of Fuzzy Mathematical Programming Approach for Solving Aggregate Production Planning", WCCI 2012 IEEE World Congress on Computational Intelligence, Brisbane, Australia, 2012.

[23] M.K. Omar, M. M. Jusoh, M. Omar, "Developing a Master Production Schedule Using Fuzzy Mixed Integer Linear Programming", Conference on Applied Mathematics and Computational Methods, Rhode Island, Grece, 2013, pp. 215-220

[24] Michael L. Pinedo, "Scheduling. Theory, Alghoritms and Systems", Third Edition, Springer, 2008

[25] D. Opresnik, M. Fiasché, M. Taisch, M Hirsch, "An evolving fuzzy inference system for extraction of rule set for planning a product-service strategy", Information Technology and Management, 2015, DOI 10.1007/s10799-015-0242-4

[26] M. Fiasché, A.Verma, M. Cuzzola, F.C. Morabito G. Irrera, "Incremental- Adaptive- Knowledge Based- Learning for Informative Rules Extraction in Classification Analysis of aGvHD", IFIP Advances in Information and Communication Technology, Volume 363 AICT, Issue PART 1, 2011, Pages 361-371. DOI: 10.1007/978-3-642-23957$1 \_41$

[27] M. Fiasché, "A quantum-inspired evolutionary algorithm for optimization numerical problems". In LNCS : Vol. 7665, 2012, ICONIP 2012, Part III (pp. 686-693), doi: 10.1007/978-3-642-34487-9_83 